\title{
Amine-Functionalized Graphene Quantum Dots for Fluorescence-Based Immunosensing of Ferritin
}

\section{Mayank Garg ${ }^{1,2}$, Neelam Vishwakarma1, Amit L. Sharma ${ }^{1,2 *}$, Suman Singh ${ }^{1,2^{*}}$}

${ }^{1}$ CSIR- Central Scientific Instruments Organisation, Sector 30-C, Chandigarh-160030, India

${ }^{2}$ Academy of Scientific and Innovative Research (AcSIR), Ghaziabad-201002, India

*Corresponding author: ssingh@csio.res.in, amitsharma csio@csio.res.in

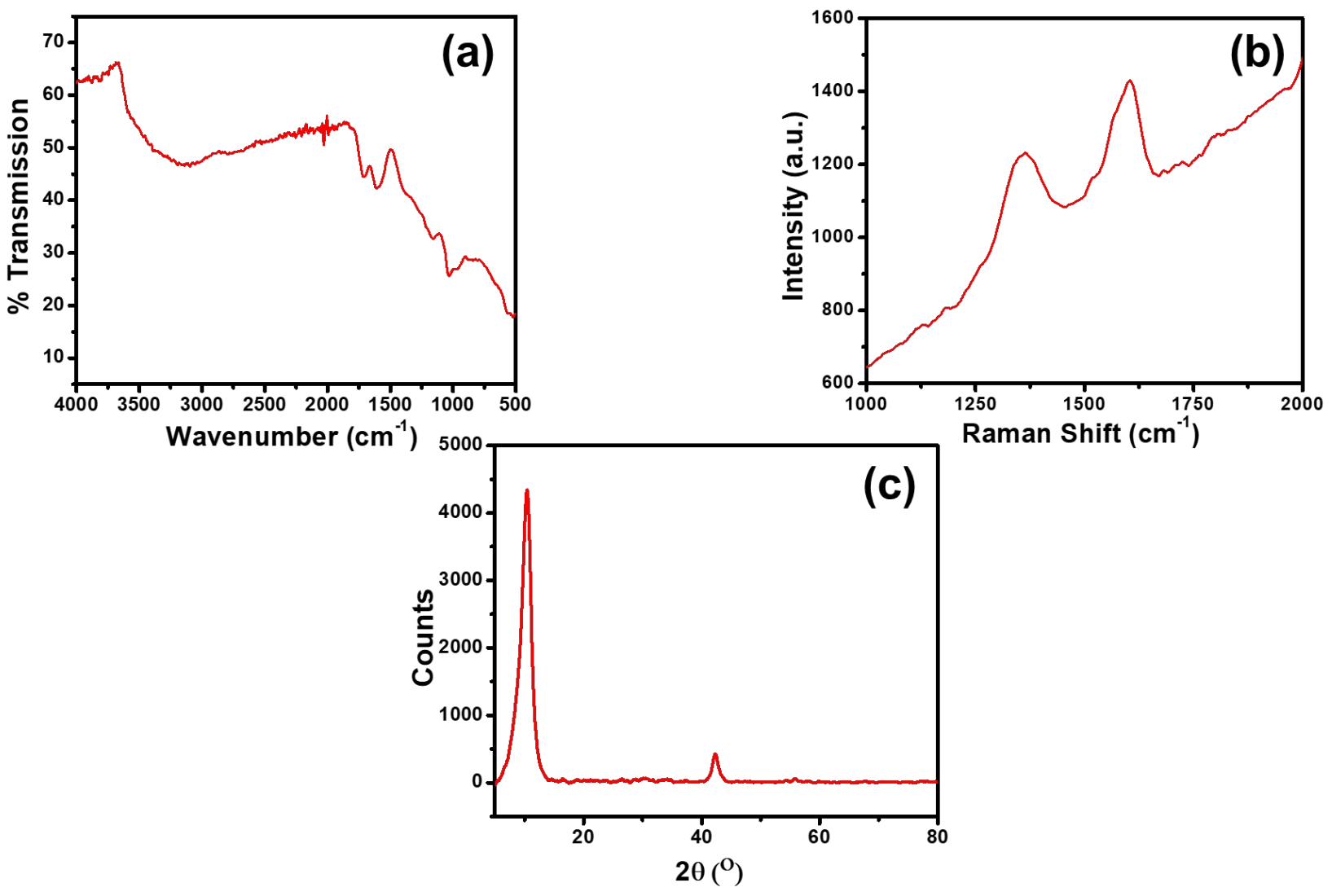

Fig. S1: (a) FTIR, (b) Raman spectra and (c) XRD pattern of graphene oxide 


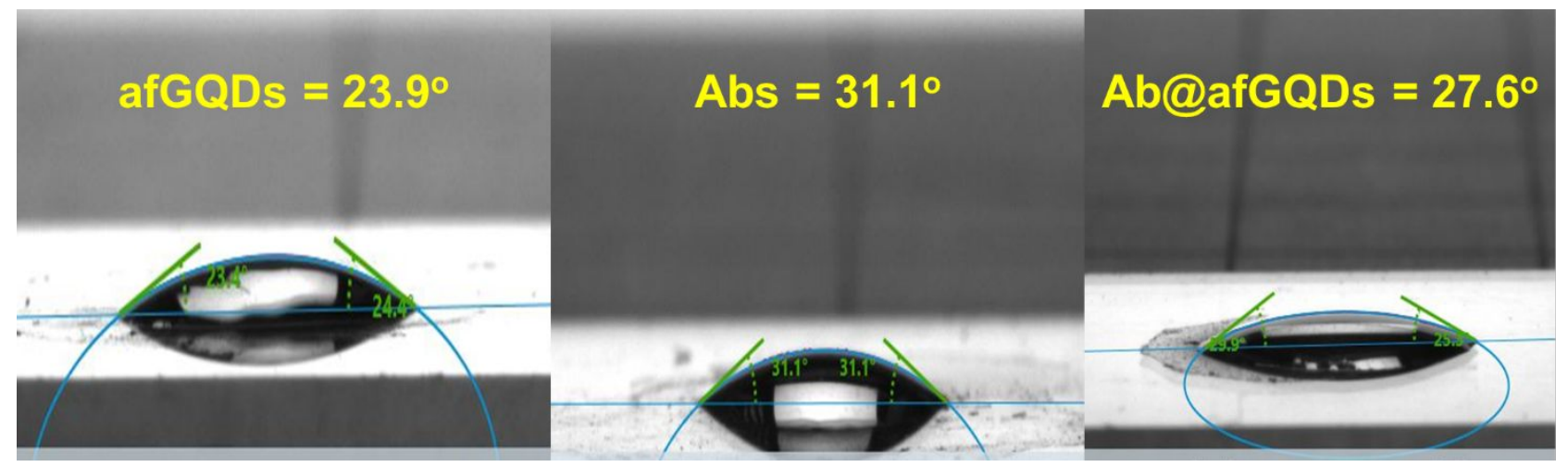

Fig. S2: Contact angle measurements of afGQDs, antibodies (Abs) and antibody immobilized on afGQDs (Ab@afGQDs)

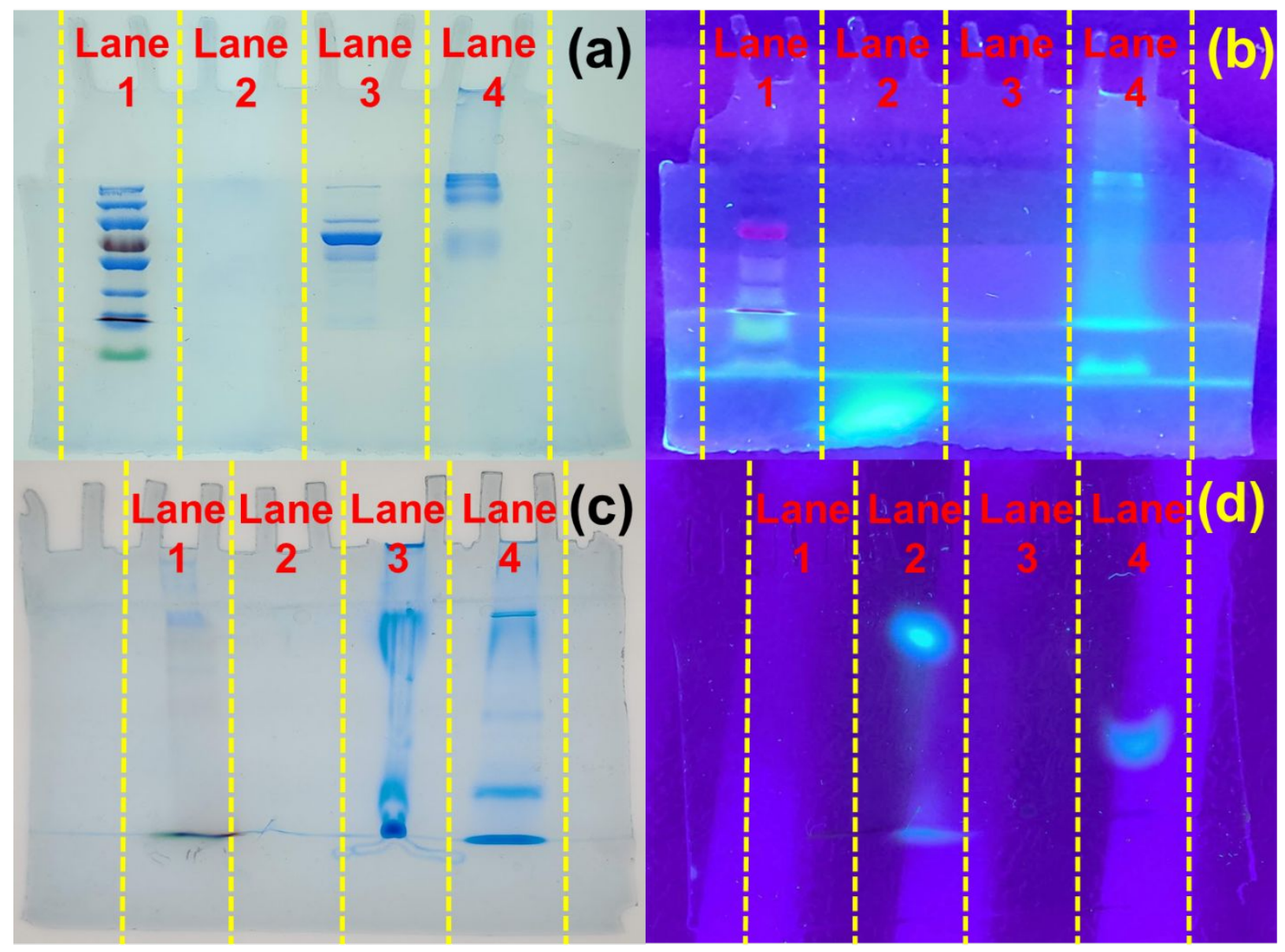

Fig. S3: (a) Image showing the bands obtained after SDS-PAGE. Lane 1: Protein Ladder; Lane 2: afGQDs; Lane 3: antibodies; Lane 4: Ab@afGQDs under visible light and (b) showing the same bands under UV light (c) Image showing the bands obtained after Native-PAGE. Lane 1: 
Protein Ladder; Lane 2: afGQDs; Lane 3: antibodies; Lane 4: Ab@afGQDs under visible light and (d) showing the same bands under UV light [UV image enhanced: Brightness: $0 \%$ (Normal), Contrast: $-40 \%$ for better visualization]

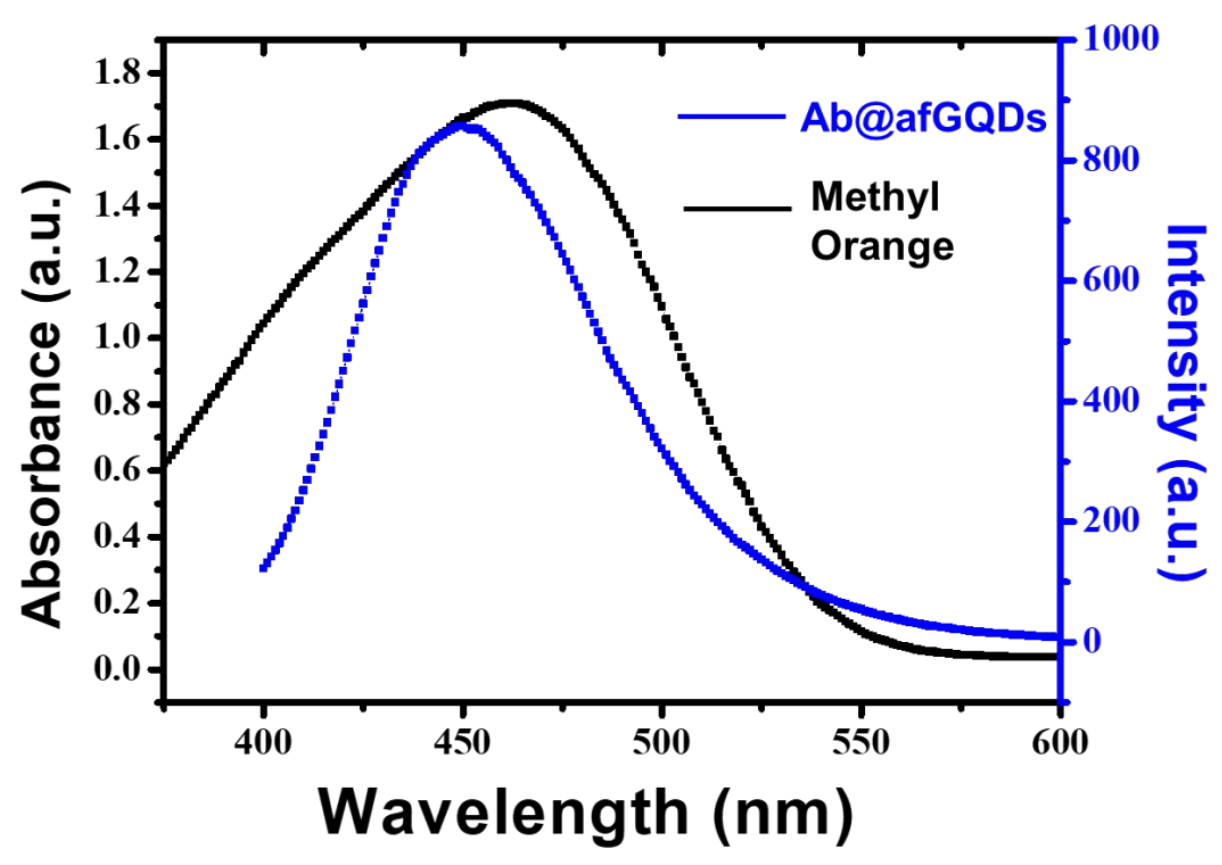

Fig. S4: Spectral overlap of absorption spectrum of methyl orange and emission spectrum of Ab@afGQDs 


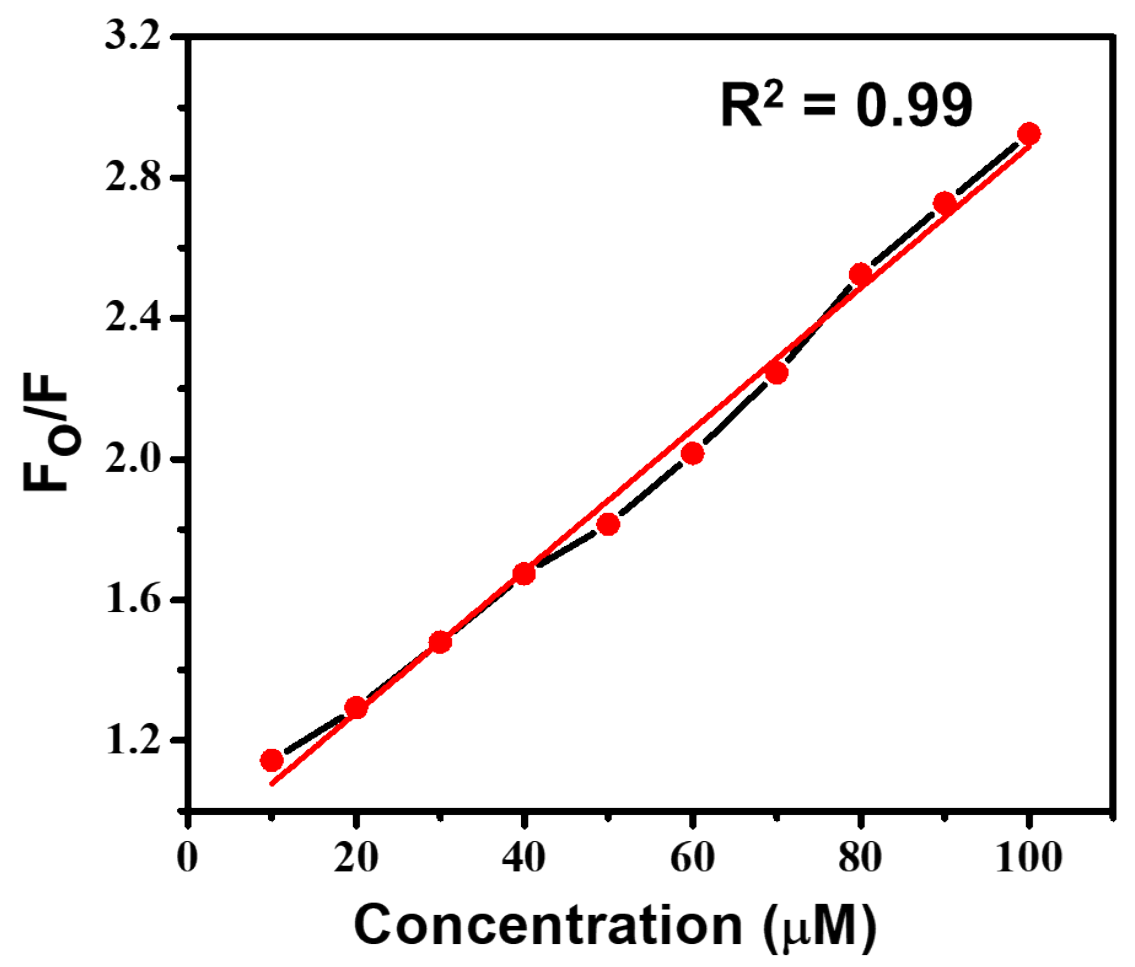

Fig. S5: Stern-Volmer Plot of $A b @ a f G Q D s$ in the presence of increasing methyl orange concentrations $\left[F_{o}\right.$ and $F$ is the fluorescence intensity of $A b @$ afGQDs in the absence and presence of methyl orange respectively]
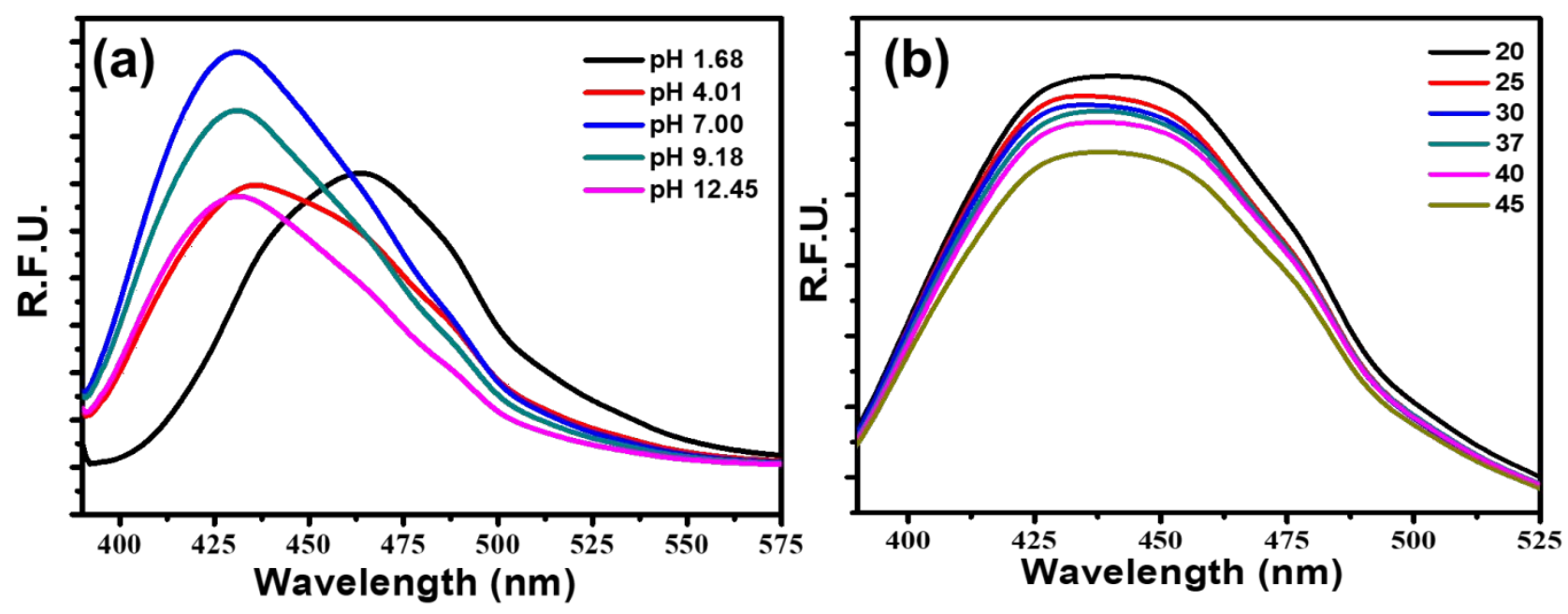

Fig. S6: (a) Effect of pH and (b) Effect of temperature [R.F.U.: Relative Fluorescence Units] 


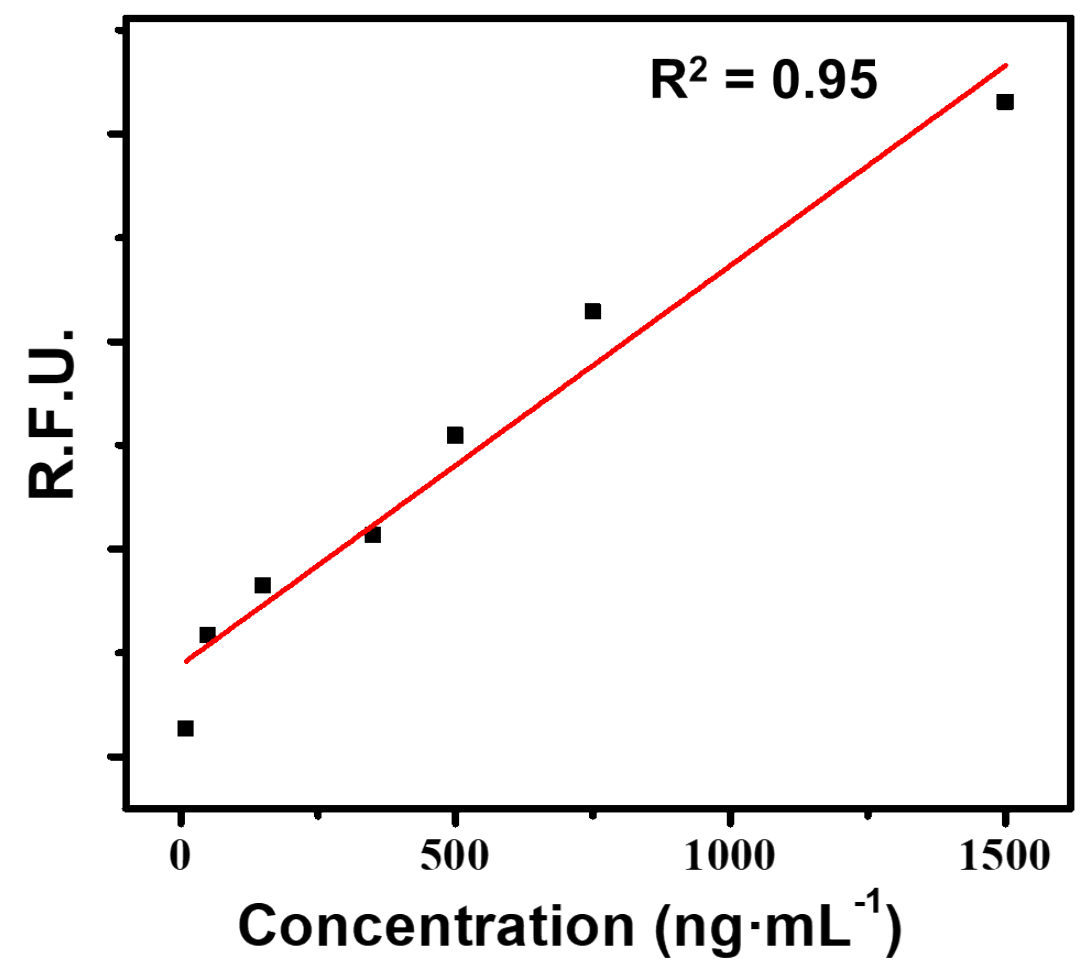

Fig. S7: Calibration curve for ferritin sensing in serum samples [R.F.U.: Relative Fluorescence Units] 